\title{
Posttraumatic Intracranial Tuberculous Subdural Empyema in a Patient with Skull Fracture
}

\author{
Jiha Kim, M.D., ${ }^{1}$ Choonghyo Kim, M.D., ${ }^{1}$ Young-Joon Ryu, M.D., ${ }^{2}$ Seung Jin Lee, M.D. \\ Departments of Neurosurgery, ${ }^{1}$ Pathology, ${ }^{2}$ Kangwon National University Hospital, Kangwon National University School of Medicine, Chuncheon, Korea
}

\begin{abstract}
Intracranial tuberculous subdural empyema (ITSE) is extremely rare. To our knowledge, only four cases of microbiologically confirmed ITSE have been reported in the English literature to date. Most cases have arisen in patients with pulmonary tuberculosis regardless of trauma. A 46-year-old man presented to the emergency department after a fall. On arrival, he complained of pain in his head, face, chest and left arm. He was alert and oriented. An initial neurological examination was normal. Radiologic evaluation revealed multiple fractures of his skull, ribs, left scapula and radius. Though he had suffered extensive skull fractures of his cranium, maxilla, zygoma and orbital wall, the sustained cerebral contusion and hemorrhage were mild. Eighteen days later, he suddenly experienced a general tonic-clonic seizure. Radiologic evaluation revealed a subdural empyema in the left occipital area that was not present on admission. We performed a craniotomy, and the empyema was completely removed. Microbiological examination identified Mycobacterium tuberculosis (M.tuberculosis). After eighteen months of anti-tuberculous treatment, the empyema disappeared completely. This case demonstrates that tuberculosis can induce empyema in patients with skull fractures. Thus, we recommend that $M$. tuberculosis should be considered as the probable pathogen in cases with posttraumatic empyema.
\end{abstract}

Key Words : Empyema $\cdot$ Skull fracture $\cdot$ Trauma $\cdot$ Tuberculosis.

\section{INTRODUCTION}

Subdural empyema is a rare intracranial infection that usually arises as a complication of meningitis in infants and young children and as a complication of paranasal sinusitis, otitis media, or mastoiditis in older children and adults ${ }^{3,6,11)}$. The organism responsible for a subdural empyema depends on the origin of the infection ${ }^{3)}$. Aerobic and anaerobic streptococci, Haemophilus influenzae, Staphylococcus aureus, and Staphylococcus epidermidis are the most commonly identified organisms ${ }^{3)}$. Mycobacterium tuberculosis (M.tuberculosis) is an extremely rare pathogen in subdural empyema ${ }^{2,3,11,12)}$. To date, only four cases of microbiologically confirmed intracranial tuberculous subdural empyema (ITSE) have been reported in the English literature ${ }^{2,3,11,12)}$.

Neurotuberculosis constitutes approximately $5-15 \%$ of all cases of extra-pulmonary tuberculosis ${ }^{1,5,7)}$. Although tuberculous meningitis and parenchymal granulomas cause most cases of intracranial neurotuberculosis ${ }^{1,3,5,7,12)}$, ITSE is an extremely rare condition ${ }^{2,3,11-13)}$. Most of the cases have been associated with pulmonary tuberculosis, which was thought to be the origin of hematogenous dissemination ${ }^{2,3,12)}$.
To our knowledge, posttraumatic ITSE with skull fracture has not yet been reported. Our patient experienced a tuberculous empyema after a head trauma that resulted in skull fracture. Although an inactive pulmonary tuberculous lesion was identified on his chest computed tomography (CT) scan, there was no subdural empyema immediately after the skull fracture. Therefore, our case is unique among the previously reported cases because the skull fracture may have affected the occurrence of ITSE.

\section{CASE REPORT}

A 46-year-old man presented to the emergency department with head trauma. He had sustained a fall while working at an approximately 6-meter-high location. Upon arrival at our hospital, he complained of severe pain in his head, face, chest, and left arm. He was alert and oriented, and the initial neurological examination was normal. Although there were some abrasions, he had no lacerations or deep wounds on his head and face. CT scan revealed multiple fractures involving his skull, facial bones, ribs, left scapula and left distal radius. Although the skull and

- Received : December 23, 2014 • Revised : April 9, 2015 •Accepted : April 17, 2015

- Address for reprints : Seung Jin Lee, M.D.

Department of Neurosurgery, Kangwon National University Hospital, 156 Baengnyeong-ro, Chuncheon 24289, Korea

Tel : +82-33-258-2410, Fax : +82-33-251-0374, E-mail : rabbit3540@empas.com

- This is an Open Access article distributed under the terms of the Creative Commons Attribution Non-Commercial License (http://creativecommons.org/licenses/by-nc/3.0) which permits unrestricted non-commercial use, distribution, and reproduction in any medium, provided the original work is properly cited. 
Fig. 1. Brain CT scan performed on the first day shows a skull fracture $(A)$ and minimal cerebral contusion and intracranial hemorrhage (B).
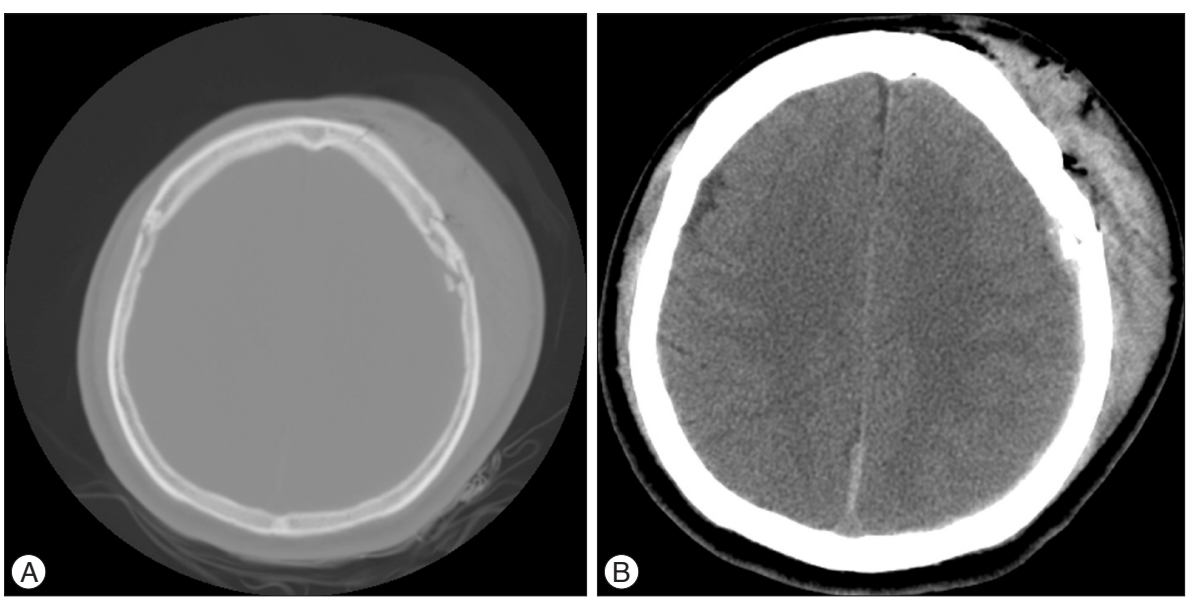

Fig. 2. A : Preoperative brain CT scan performed on the eighteenth day shows left fronto-temporal and left occipital fluid collections. B : Preoperative axial MRI, T1-weighted gadolinium-enhanced images shows the empyema in the left occipital region.
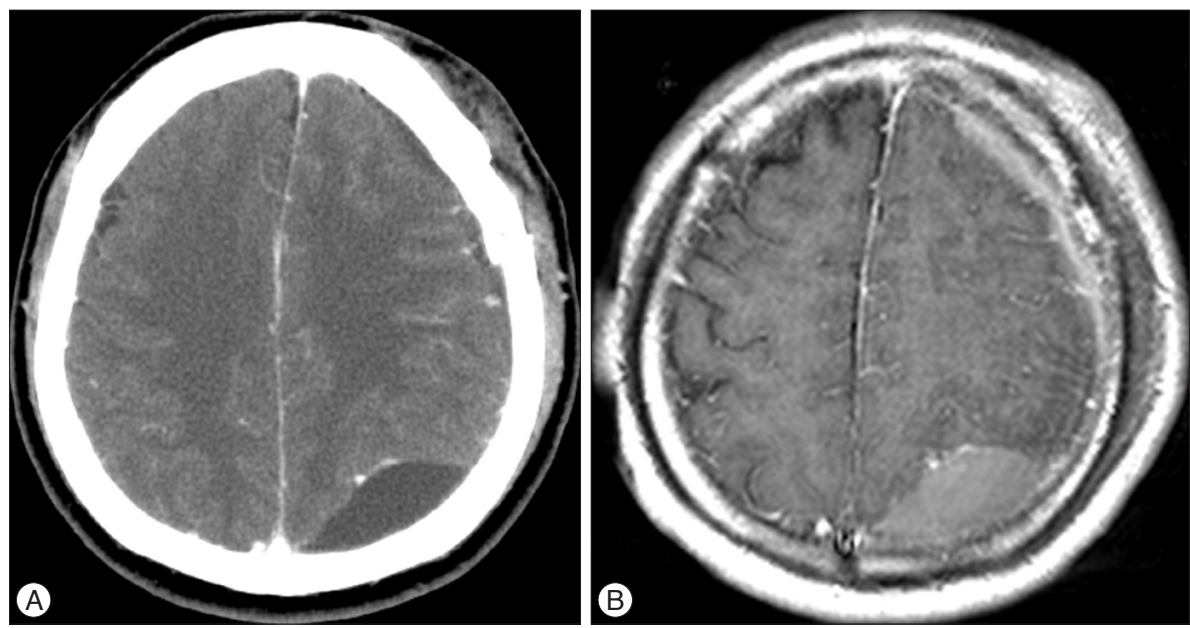

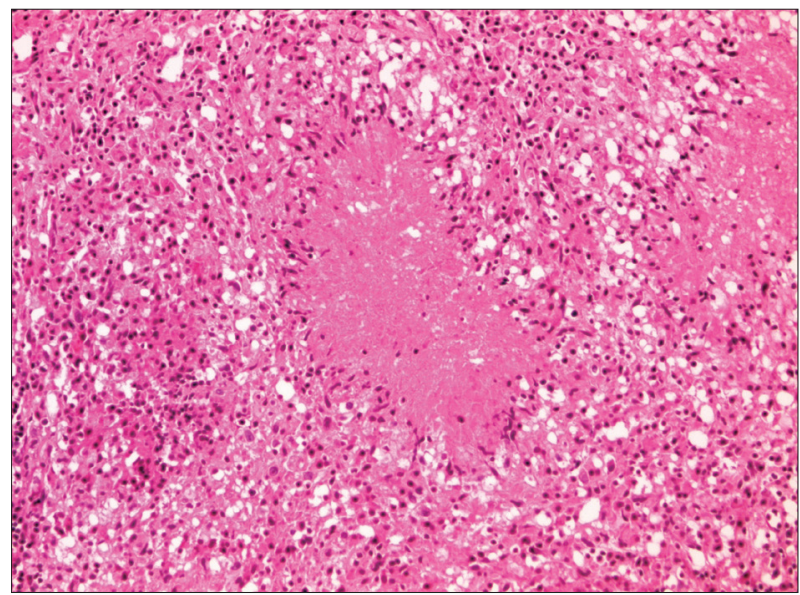

Fig. 3. Photomicrograph of sections used for histopathological examination shows central caseous necrosis and palisading epithelioid cells (hematoxylin and eosin staining, original magnification $\times 200$ ).

facial bone fractures were extensive in the left fronto-temporal cranium, left maxilla, left zygoma and left orbital wall, the cerebral contusion and intracranial hemorrhage were mild (Fig. 1). Although there was pneumocephalus in the left frontal area, he did not experience cerebrospinal fluid (CSF) rhinorrhea. We performed conservative management after inserting a chest tube to drain the hemothorax.

Eighteen days later, he suddenly experienced a general tonicclonic seizure. The follow-up brain CT scan revealed fluid collection in the left fronto-temporal skull fracture site and in the left occipital area that had not been present on admission (Fig. 2A). Magnetic resonance imaging (MRI) demonstrated strong enhancement of the dura mater surrounding the left occipital fluid collection, and the fluid was suspected to be a subdural empyema (Fig. 2B).

We performed a craniotomy, and both the left fronto-temporal fluid collection and the left occipital subdural empyema were completely removed. The occipital empyema was thick and dark brownish, making it distinct from the clear fluid in the left fronto-temporal area. There was no traumatic lesion in the occipital area, whereas multiple dural tears were found intraoperatively at the left fronto-temporal skull fracture site.

Polymerase chain reaction (PCR) analysis confirmed M. tuberculosis in the empyema. The histopathological examination produced the same result, showing multifocal granulomatous inflammation with extensive caseous necrosis and some acidfast bacilli (AFB) (Fig. 3). Only M. tuberculosis bacilli were identified without co-infection with other microorganisms. Our patient denied any previous history of tuberculosis elsewhere in 


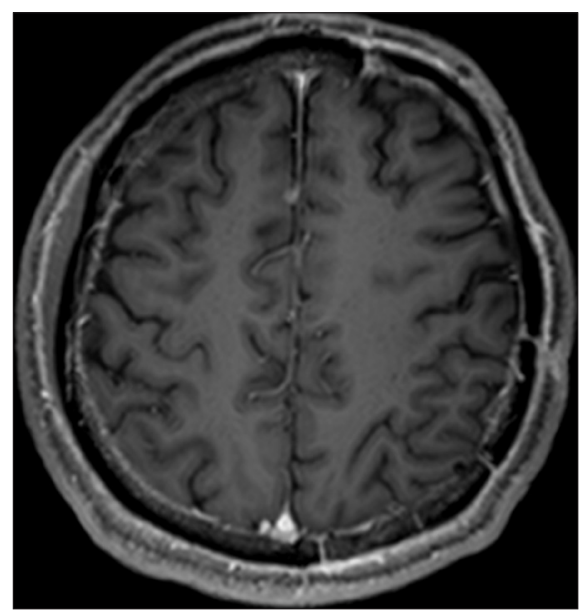

Fig. 4. Axial MRI performed six months after the operation, T1-weighted gadolinium-enhanced image shows complete disappearance of the left occipital empyema.

his body and presented no clinical evidence of pulmonary tuberculosis. Moreover, sputum AFB stain, culture and PCR tests were all negative. However, we found calcified nodules on a chest $\mathrm{CT}$ scan, suggesting inactive tuberculosis.

We administered anti-tuberculous treatment for eighteen months. A follow-up MRI performed six months later showed the complete disappearance of the subdural empyema (Fig. 4).

\section{DISCUSSION}

\section{Association with pulmonary tuberculosis}

Most cases of ITSE are closely related to pulmonary tuberculosis. To our knowledge, four cases of ITSE have been published thus $\mathrm{far}^{2,3,11,12)}$. Two of them had pulmonary tuberculosis, which was thought to be the origin of the neurotuberculosis ${ }^{3,12}$. Another one of the cases had a history of exposure to an active case of pulmonary tuberculosis ${ }^{2}$. Whereas only one-third of tuberculous brain abscesses are accompanied by pulmonary tuberculosis ${ }^{1)}$, ITSE seems to be associated more frequently with a pulmonary focus.

Cayli et al. ${ }^{3)}$ reported a 1-year-old boy with ITSE whose infection resulted from hematogenous dissemination of tuberculous bacilli from the primary lesion in the lung. van Dellen et al. ${ }^{12)}$ described ITSE that started as tuberculous osteitis of the cranium, which had originated from pulmonary tuberculosis. Despite a 5-year history of treatment for pulmonary tuberculosis, he had poor compliance in maintaining anti-tuberculous treatment. It is the only case of ITSE that arose in the adult population. Banerjee et al. ${ }^{2}$ also reported a 12 -year-old boy with ITSE. Although the presence of pulmonary tuberculosis was not described, he had a history of exposure to an active case of pulmonary tuberculosis (his father ${ }^{2}$. Long-term exposure to pulmonary tuberculosis is likely to cause the inhalation of aerosolized droplets contaminated with tuberculous bacilli. Thus, the hematogenous dissemination of tuberculous bacilli from the lung to the subdural space was considered the probable patho- genic process of his ITSE.

Our patient had inactive pulmonary tuberculosis that was identifiable on chest $\mathrm{CT}$ scan, although the sputum AFB culture and PCR test were negative. However, we are not sure that his pulmonary tuberculosis was the source of the subdural empyema as in the aforementioned cases. Although his latent pulmonary lesion may be the source of the empyema, it is very difficult to explain what caused the inactive pulmonary tuberculosis to become active immediately after he sustained the skull fracture. Moreover, the subdural empyema was not present on the initial brain CT scan performed immediately after the trauma, but it was easily detected eighteen days later (Fig. 1B, 2A). Therefore, we believe that the empyema arose after the head trauma, and the effects of the pulmonary tuberculosis were very limited in our ITSE case. We think that the trauma and the resultant skull fracture must have facilitated the development of a subdural empyema by destroying physiological barriers such as the dura, arachnoid or blood-brain barrier, offering the pathogen an entry point. We also believe that the basal skull fracture caused pathologic communication between the cranial cavity and the open air, as evidenced by the pneumocephalus detected on CT scan. Moreover, several dural tears were identified intraoperatively. The dura is an effective barrier to the contiguous spread of all infections ${ }^{2,12}$; therefore, the dural tears in our case might have allowed the bacilli to pass into the subdural space and facilitated direct subdural spreading.

\section{Basal skull fracture}

Basal skull fractures are closely associated with meningitis with or without CSF leakage ${ }^{8-10)}$. The reported incidence of meningitis following basal skull fracture varies from 0.4 to $17.8 \%{ }^{9}$. Dural tears are common because of the firm adherence of the dura to the cranial base ${ }^{8)}$, and CSF rhinorrhea is present in less than $10 \%$ of cases with anterior fossa fractures ${ }^{9}$. Our patient had multiple fractures in the anterior skull base and temporal cranium. In addition, several dural tears were identified intraoperatively near the fracture site. Although our patient had pneumocephalus on the initial CT scan, CSF leakage was not observed.

Meningitis may also occur in the absence of an apparent CSF ${ }^{l} \mathrm{eak}^{8}$. The reason is unclear, but it may be related to the intermittent nature of CSF leakage or its spontaneous remission ${ }^{8)}$. Eftekhar et al. ${ }^{4)}$ noted that pneumocephalus significantly increases the risk of meningitis and should therefore be considered equivalent to CSF leakage. Additionally, Scholsem et al. ${ }^{8)}$ recommended surgical repair for an enlarging pneumocephalus and CSF leakage. However, most of the available information about posttraumatic meningitis is mainly for bacterial infections. There is a paucity of literature about tuberculous meningitis that stems from its rarity, although tuberculous meningitis shares some characteristics with bacterial meningitis. We believe that the current case is an important addition to the literature on ITSE, whose clinical characteristics have not yet been elucidated. 


\section{Identification of $M$. tuberculosis}

M. tuberculosis is notoriously difficult to culture, particularly after treatment has been initiated ${ }^{12}$. In our case, the AFB culture was negative even though anti-tuberculous treatment had not yet been commenced. Although we failed to culture $M$. $t u$ berculosis, the diagnosis was confirmed by a PCR test and histopathological examination. Some authors have reported co-infections of tuberculosis with other pathogens such as Staphylococcus aureus in their ITSE cases ${ }^{11,12}$. However, we did not identify any other pathogens by culturing except $M$. tuberculosis. Therefore, we are sure that the subdural empyema stemmed entirely from M. tuberculosis without the involvement of other bacterial pathogens.

Some physicians recommend the initiation of anti-tuberculous treatment on an empirical basis, even in the absence of laboratory confirmation of the diagnosis, if neurotuberculosis is suspected $^{12)}$. With the advent of human immunodeficiency virus (HIV) and acquired immunodeficiency syndrome, more cases of neurotuberculosis are being diagnosed ${ }^{1}$. However, our patient was immunocompetent, HIV negative and had no other general risk factors for infection. Thus, we did not suspect tuberculous empyema because it was too rare to be included in a differential diagnosis.

\section{CONCLUSION}

Our patient experienced a tuberculous empyema after head trauma that resulted in a skull fracture. Our case is very unique in that $M$. tuberculosis caused a subdural empyema in a patient who had a skull fracture after head trauma. We recommend that $M$. tuberculosis should be considered as a likely pathogen in posttraumatic empyema in patients with skull fractures.

\section{References}

1. Ansari MK, Jha S: Tuberculous brain abscess in an immunocompetent adolescent. J Nat Sci Biol Med 5 : 170-172, 2014

2. Banerjee AD, Pandey P, Ambekar S, Chandramouli BA : Pediatric intracranial subdural empyema caused by Mycobacterium tuberculosis--a case report and review of literature. Childs Nerv Syst 26 : 1117-1120, 2010

3. Cayli SR, Onal C, Koçak A, Onmuş SH, Tekiner A : An unusual presentation of neurotuberculosis : subdural empyema. Case report. J Neurosurg 94 : 988-991, 2001

4. Eftekhar B, Ghodsi M, Nejat F, Ketabchi E, Esmaeeli B : Prophylactic administration of ceftriaxone for the prevention of meningitis after traumatic pneumocephalus : results of a clinical trial. J Neurosurg 101 : 757-761, 2004

5. Katti MK : Pathogenesis, diagnosis, treatment, and outcome aspects of cerebral tuberculosis. Med Sci Monit 10 : RA215-RA229, 2004

6. Lee SK, Kim SW : Fatal subdural empyema following pyogenic meningitis. J Korean Neurosurg Soc 49 : 175-177, 2011

7. Madhavan K, Widi G, Shah A, Petito C, Gallo BV, Komotar RJ : Tuberculoma of the brain with unknown primary infection in an immunocompetent host. J Clin Neurosci 19: 1320-1322, 2012

8. Scholsem M, Scholtes F, Collignon F, Robe P, Dubuisson A, Kaschten B, et al. : Surgical management of anterior cranial base fractures with cerebrospinal fluid fistulae : a single-institution experience. Neurosurgery 62 : 463-469; discussion 469-471, 2008

9. Servais L, Fonteyne C, Christophe C, Prudhon V, Brihaye P, Biarent D, et al. : Meningitis following basal skull fracture in two in-line skaters. Childs Nerv Syst $21:$ 339-342, 2005

10. Sonig A, Thakur JD, Chittiboina P, Khan IS, Nanda A : Is posttraumatic cerebrospinal fluid fistula a predictor of posttraumatic meningitis? A US Nationwide Inpatient Sample database study. Neurosurg Focus 32 : E4, 2012

11. Turel MK, Moorthy RK, Rajshekhar V : Multidrug-resistant tuberculous subdural empyema with secondary methicillin-resistant Staphylococcus aureus infection : an unusual presentation of cranial tuberculosis in an infant. Neurol India $60: 231-234,2012$

12. van Dellen A, Nadvi SS, Nathoo N, Ramdial PK : Intracranial tuberculous subdural empyema : case report. Neurosurgery 43 : 370-373, 1998

13. Vijayakumar B, Sarin K, Mohan G : Tuberculous brain abscess and subdural empyema in an immunocompetent child : significance of AFB staining in aspirated pus. Ann Indian Acad Neurol 15 : 130-133, 2012 